Research Article

Arome Junior Gabriel*, Boniface Kayode Alese, Adebayo Olusola Adetunmbi, Olumide Sunday Adewale, and Oluwafemi Abimbola Sarumi

\title{
Post-Quantum Crystography System for Secure Electronic Voting
}

https://doi.org/10.1515/comp-2019-0018

Received Aug 31, 2018; accepted Sep 09, 2019

\begin{abstract}
Security (privacy, confidentiality and integrity) of pre-electoral, electoral and post electoral phases of the electioneering process is fundamental to the success of Electronic Voting (E-Voting) Systems. Crystography, which is the combination of cryptography and steganography could be a fitting 'tool kit' for enhancing the security of sensitive election-related information transmitted over public networks, thereby also ensuring free, fair and credible election/voting. Most of the existing secure e-voting systems are based on public key cryptographic schemes like RSA and Elliptic Curve Cryptography (ECC), whose security depends on the difficulty of solving Integer Factorization Problem (IFP) and Discrete Logarithm problem (DLP) respectively. However, techniques for solving IFP and DLP problems, improves continually. One of such is the quantum algorithm discovered by Peter Shor in 1994, which can solve both IFP and DLP problems in polynomial time. Consequently, the existence of quantum computers in the range of 1000 bits would spell doom to systems based on those problems. This paper presents the development of a new crystographic system that combines Post Quantum Cryptography with steganography to ensure that the security of e-voting is maintained both in classical computing era as well as post-quantum computing era. Our experiments' results shows that our proposed system performed better than existing ones.
\end{abstract}

Keywords: post-quantum computing, cryptography, electronic-voting, information security, steganography

\footnotetext{
^Corresponding Author: Arome Junior Gabriel: Department of Cyber Security Science, Federal University of Technology, Akure, Nigeria; Email: ajgabriel@futa.edu.ng

Boniface Kayode Alese: Department of Cyber Security Science, Federal University of Technology, Akure, Nigeria;

Email: bkalese@futa.edu.ng

Adebayo Olusola Adetunmbi: Department of Computer Science, Federal University of Tenology, Akure, Nigeria;

Email: aoadetunmbi@futa.edu.ng
}

\section{Introduction}

Modern Information Technology (IT) has no doubt transformed the entire world into a "Global Village", and the explosive increase in the use of IT in almost all aspects of life has yielded several benefits ranging from improved efficiency and expediency with reduced costs. An assessment of existing literatures reveals that IT has been used to provide different electronic solutions in education [1, 2], health [3], governance [4, 5], businesses [6], communication [7], shopping [8], human resource management $[9,10]$, wheather forecasting/prediction [11] and even in democratic decision making [12-15]. The security (privacy, confidentiality and integrity) of information while in storage and even in transit during their respective e-service implementation is essential to the success rate of all these application areas [16, 17].

E-voting is defined as the use of electronic technologies to aid voting (at least the casting of votes) procedures in a decision making scenario. It is a serious issue of concern in governance especially in developing nations where electronic election is still an emerging field [12].

E-voting requires the use of automated voting equipments for voter's registration, ballot casting and counting, as well as ballot recording in a trustable way [18].

The use of electronic systems in the voting procedure comes with several advantages over the traditional paperbased voting. For instance, e-voting leads to increased participation of citizens in democratic governance, it also leads to substantial reduction in costs, as the materials required for printing and distributing ballots as well as the manpower required to govern poll sites are considerably reduced. E-voting provides for flexibility, since it can be adapted to support several languages. E-voting en-

\footnotetext{
Olumide Sunday Adewale: Department of Computer Science, Federal University of Technology, Akure, Nigeria;

Email: adewale@futa.edu.ng

Oluwafemi Abimbola Sarumi: Department of Computer Science, Federal University of Technology, Akure, Nigeria; Email: oasarumi@futa.edu.ng

Ә Open Access. (C 2019 A. Junior Gabriel et al., published by De Gruyter. 
sures greater speed and accuracy of votes counting. In evoting, the automated step-by-step processes involved ensures gross reduction in the number of rejected or poorly casted votes, and even eliminates or reduce the possibility of election fraud especially in developing countries [19].

E-voting systems must satisfy security requirements such as confidentiality, integrity, authentication and verifiability/non-repudiation, in order to effectively and successfully provide the aforementioned advantages [15].

Different information security mechanisms, including steganography, watermarking and data encryption schemes have been proposed for the enforcement of security in e-voting systems. These data encryption schemes like Data Encryption Standard (DES), Triple DES (3DES) and Advanced Encryption Standard (AES), have been found inadequate and not so efficient in the encryption of large volume of digital data [20, 21]. Moreover, encryption can arouse the suspicion of an attacker or eavesdropper. These challenges can be addressed by complementing data encryption with data hiding. Information hiding has been used to enhance security level of data encryption systems. Although, the main motivation behind information hiding is concerns over copyright, such as audio, video and other works available in digital form, Steganography (information hiding) have been employed in military communication systems. Similar modus operandi is being used in some mobile phone systems as well as schemes proposed for digital elections.

In [22], the authors carried out an extensive review of existing cryptographic, steganographic as well as steganocryptographic models for ensuring security in e-voting systems. They formulated a conceptual framework for secure e-voting and proposed a model for secure e-voting system.

Since cryptography has a serious flaw in that, sending encrypted data over open networks could arouse the awareness of eavesdroppers or attackers, steganography, which in contrast ensures covert (concealed) communication, could be combined with it, as proposed in some literatures. The other challenge however, is that, most existing cryptographic schemes are susceptible to quantum attacks. This means if after a while, quantum computers become available in commercial quantity, then there will be serious reduction in the work-factor required to break these conventional cryptographic schemes, and that could spell serious danger to secure evoting systems that depends on conventional cryptography. In this present paper, we propose the combination of steganography and postquantum cryptography, in a 2-layer security framework called post-quantum crystographic secure e-voting framework. This chemistry is expected to provide for stronger ballot protection and preservation of the electoral integrity from an adversary.

Although various security approaches such as biometrics, access control, cryptography, steganography or even watermarking have been proposed in the academic research community for secure e-voting, none of them have been able to completely eliminate the security shortcomings of current e-voting systems. Crystography can be a more suitable solution to security problems of e-voting.

The term "Crystography" refers to the combination of cryptography and steganography for the purpose of enhancing the security of communications, usually involving; images, text or even voice over public networks [21, 23].

Cryptography is the study of mathematical techniques related to aspects of information security such as confidentiality, data integrity, entity authentication, and data origin authentication [16, 24]. It protects information by converting it into an incomprehensible form. Cryptography ensures confidential transmission over a public network. The original text, or plaintext, is transformed into a scrambled equivalent called cipher-text using an encryption algorithm. Only authorized persons who have a secret key can decipher (decrypt) the cipher-text into plaintext [20]. This is a huge necessity for electronic systems, especially in the face of inccreasing cases of cyber crime occurences [25].

\section{Research motivation}

Public key cryptography is used in e-commerce for authentication (electronic signatures) and secure communication (encryption). RSA and ECC (elliptic curve cryptosystems) are the most widely used cryptosystems. Their security is based respectively on the problem of integer factorization and discrete logarithm. However, improvements in factorization algorithm and computation power require larger bit size in RSA key. However, larger key size makes RSA less efficient for practical applications. Although researchers have shown that ECC are more efficient as compared to RSA, the former is widely regarded as susceptible to some carefully perpetuated classical and quantum cyber-attacks [16, 20, 25]. Furthermore, Peter Shor a mathematician and scientist discovered in 1994, a polynomial time algorithm for integer factorization and computation of discrete logarithm on quantum computers [16]. This Algorithm, together with the explosive increase in processing power to be provided by quantum computers, will greatly produce reductions in the work factor required to 
solve IFP and DLP problems, and thus pose serious threats to current information security infrastructure [21, 26, 27].

To the best of my knowledge, most existing crystosystems are built around public key cryptographic schemes like RSA and ECC, whose security stems from the difficulty in solving IFP and DLP problems respectively. Thus, once quantum computers becomes available in commercial quantity, such systems can no longer be considered secure.

There is therefore a reasonable motivation to embark on the development of public key crystographic systems whose security are based on problems that are secure on both conventional and quantum computers.

Besides, studying post-quantum crypto-schemes is a worthwhile venture as it comes with a lot of benefits; firstly, it allows cryptographers to proactively deal with the challenges of post-quantum world. Secondly, it also allows a greater diversification of cryptosystems. Thirdly, it helps to greatly contribute to the process of building confidence in the security of alternative cryptosystems which will definitely span many years. Fourthly, it is best to start this process early and without the pressure of a near breakdown of cryptography, whether it comes from quantum computers or any other kind of attack.

\section{Specific objective}

The specific objective of this research is to develop a robust two-layer classical and quantum attack resistant security architecture for secure electronic voting. The idea is basically to ensure adequate privacy and confidentiality of information about voters and their votes especially as it is transferred over enterprise network.

\section{Our Secure E-Voting Framework}

\subsection{System design}

In this paper, we illustrate the problem of secure electronic voting in terms of the popular prisoners' problem, where $\mathrm{Al}$ ice and Bob are two inmates who desire to communicate in order to devise an escape plan. They must however, ensure that all their communication does not stir-up in any way, the suspicion of Wendy the warden, who, on discovering, will put them in solitary confinement.

In our secure e-voting framework, we have Bob (the sender) representing client systems stationed at voter registration/voting centers or units. A client system wishes to securely send a secret message $M$ (that is, registration details of voters collected at the point of registration, as well as their votes) to Alice (the receiver), representing the main server system /control center, resident at the National Electoral Commission headquarter office or some remote location: in order to do this, Bob first encrypts the message using a multivariate quadratic polynomial encryption algorithm to produce a cipher-text, $Y$. He then chooses a cover image $C$. The steganography algorithm identifies $C$ 's redundant bits (i.e., those that can be modified without arousing Wendy's suspicion), and then the embedding process creates a stego-image, $S$ by replacing these redundant bits with data from the cipher-text, $Y$.

The resultant stego-image, $S$ is transmitted over a public channel (monitored by Wendy) and is received by Alice only if Wendy has no suspicion on it. Once Alice receives $S$, she can get $M$ via the extraction process as shown on the right hand side of the diagram in Figure 1.

The embedding process represents the critical task for a steganograph y system since $S$ must be as similar as possible to $C$ for avoiding Wendy's intervention (Wendy acts for the eavesdropper).

Figure 1 shows that our secure e-voting system is made up of two modules; the Cryptography module and the Steganography module.

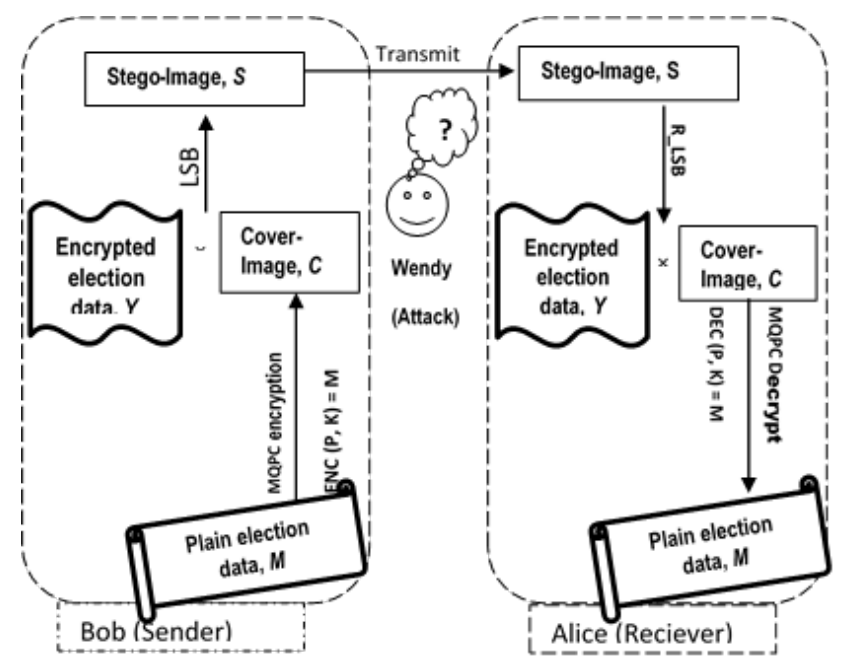

Figure 1: A Conceptual model of the secure e-voting system

The functionality of the first module is based on the problem of the simultaneous evaluation of a system of multivariate polynomial equations.

Let a polynomial vector $P$ be a system of $m$ polynomials in $n$ variables; each having a maximum degree $d \in \mathcal{N}$. 
This implies;

$$
P:=\bigcup_{i=1}^{m} p_{i}
$$

where all $p_{i}$ have the form shown in equation 2 ;

$$
\begin{aligned}
& p_{i}\left(x_{1}, \ldots, x_{n}\right):=\sum_{v \in V_{n}^{d}} \gamma_{i, v} \prod_{j=1}^{d} x_{v j} \\
& \text { for, } 1 \leq i \leq m
\end{aligned}
$$

with the coefficients $\gamma_{i, v}$ belonging to the finite field, $F$ and vectors $v$ belonging to a vector space, $V_{n}^{d}$.

Then, given $y_{1}, \ldots, y_{m} \in F$ as Field elements and multivariate polynomials $p_{i}$ as defined in equation 2 , the problem of Simultaneous Multivariate Equations (SME) is the problem of finding a solution $x \in F_{n}$ for the simultaneous system of equations in the polynomial vector $P$;

$$
\left\{\begin{array}{l}
y_{1}=p_{1}\left(x_{1}, \cdots, x_{n}\right) \\
y_{2}=p_{2}\left(x_{1}, \cdots, x_{n}\right) \\
\vdots \\
y_{m}=p_{m}\left(x_{1}, \cdots, x_{n}\right)
\end{array}\right.
$$

The Hidden Field Equations (HFE) trapdoor was deployed for encryption/decryption purposes. The HFE takes advantage of the SME problem which is considered Non deterministic Polynomial Complete (NP-complete) over finite fields. In order to avoid computational complexity that comes with higher values of the degree $d$, only multivariate polynomials of maximum order 2 are considered in this work.

The private key for the cryptographic module of the proposed system is a triple $S, P$ and $T$; where, $S$ and $T$ are affine transformations while $P$ is a private polynomial. The public key $k$ are polynomials $\left(p_{1}, \ldots, p_{n}\right)$ over $F$ with each of them depending on $n$ variables $\left(x_{1}, \ldots, x_{n}\right)$.

Encrypting a given message $m$, requires translation of such message to a vector $\left(x_{1}, \ldots, x_{n}\right)$ over $F^{n}$. Affine transformation $S$ is then applied to the vector to yield the result $x^{\prime}$. Then, in order to allow the application of the private polynomial $P \in E[x]$, using a simple correspondence between the coefficients, the result $\left(x^{\prime}\right)$, is transformed from the field $F^{n}$ to its extension $E$. This transformation yields, $y^{\prime}:=P(x)$.

Then comes the application of the other affine transformation $T$, to yield $y=\left(y_{1}, \ldots, y_{n}\right)$, which is the final output of the cryptographic module.

Furthermore, the output of the Cryptography module is fed into the Steganography module as input. In this module, the Least Significant Bit (LSB) approach is deployed. Bits of the ciphertext are directly embedded into least significant bit plane of a chosen cover image in a deterministic sequence. The cover image of size of $m \times n$ is first divided into non-overlapping blocks of $2 \times 2$ pixels $[P(x, y), P(x+1, y), P(x, y+1)$ and $P(x+1)(y+1)]$, each of which is rotated by a random degree in the range of $\{0,90,180,270\}$. One pixel is taken as the current pixel, which is then used to generate pairs with the others. The difference value for pixel pairs is calculated and used for identification of the region in which the secret data is to be embedded. The resulting stego-image was then sent across the network to the designated receiver. The receiver then extracts the cipher-text and performs decryption, to recover the original plain message.

\subsection{System implementation}

An application software was developed to simulate the secure e-voting system using NetBeans IDE 7.1.2, My Structured Query Language (MySQL) relational database management system as backend engine, Java programming Language for the coding and WAMP as the web server. The platforms for the experiment was Windows 8 Operating System running ona PC with Intel(R) Core(TM) 2 Duo processors at 4.00GHZ, and 4.00GB of RAM.

The implementation of RSA, ECC and MQPC based electronic voting systems were then tested and evaluated on Windows 8 Operating System, using the following standard metrics

- Computation time

- Size of Output

- Throughput

\section{Experiment results and discussion}

As explained earlier, we have tested the implementation of RSA, ECC and MQPC based crysto-systems and evaluated them based on the following standard metrics; Computation time, Output Size and Throughput.

\subsection{Computation time}

The Computation time here refers to the time it takes to encrypt given election data and embed the resultant ciphertext in a chosen cover-image, or the time it takes to regain the original plain-text from a given stego image. 
Table 1: Time taken in milliseconds to encrypt and embed a given voter information (text) across RSA, ECC and MQPC.

\begin{tabular}{cccc}
\hline Size of Input & RSA & ECC & MQPC \\
\hline 10 & 765 & 829 & 203 \\
20 & 750 & 453 & 78 \\
30 & 578 & 500 & 156 \\
40 & 532 & 469 & 141 \\
50 & 546 & 453 & 125 \\
60 & 610 & 516 & 156 \\
\hline
\end{tabular}

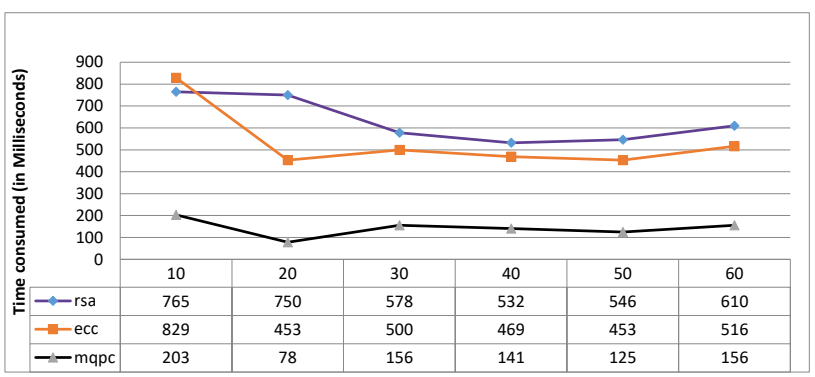

Figure 2: Performance (Forward Process) of RSA, ECC and our new MQPC crystography based e-voting systems.

Table 1 shows the time it takes (in milliseconds), to encrypt and embed a given election/voters information (text) in a selected cover-image to yield a stego-image.

The results in Table 1 were plotted as graph in Figure 2. This represents the performance of crysto-systems that are based on RSA, ECC and MQPC, with respect to time taken to encipher and then, embed a given message in a particular cover image. It can be clearly deduced that our postquantum secure e-voting system performed better than the others, as it takes lesser time to encipher a message and then, embed the resultant cipher-text in a given cover media. ECC comes next; performing better than RSA.

\subsection{Size of Output}

In any cryptographic algorithm, it is essential to understand the size of the input and the size of output. Larger size of the cipher-text compared with the Plain-text, could imply that the cipher-text is more robust against any BruteForce attack. In this work, the Output size refers to the size of the cipher-text after it has been embedded in a coverimage.

The second performance criterion used in this work is the size of the final output when the entire crystographic process is completed. Figure 3 shows the graph of the output sizes plotted against the initial plaintext sizes. It is essential to understand that the size of the input and the size
Table 2: Size of output generated after encrypting and embedding a given voter information (text) across RSA, ECC and the proposed MQPC.

\begin{tabular}{cccc}
\hline Size of Input & RSA & ECC & MQPC \\
\hline 10 & 44 & 48 & 48 \\
20 & 44 & 48 & 80 \\
30 & 44 & 96 & 128 \\
40 & 44 & 96 & 160 \\
50 & 44 & 96 & 208 \\
60 & 44 & 96 & 240 \\
\hline
\end{tabular}

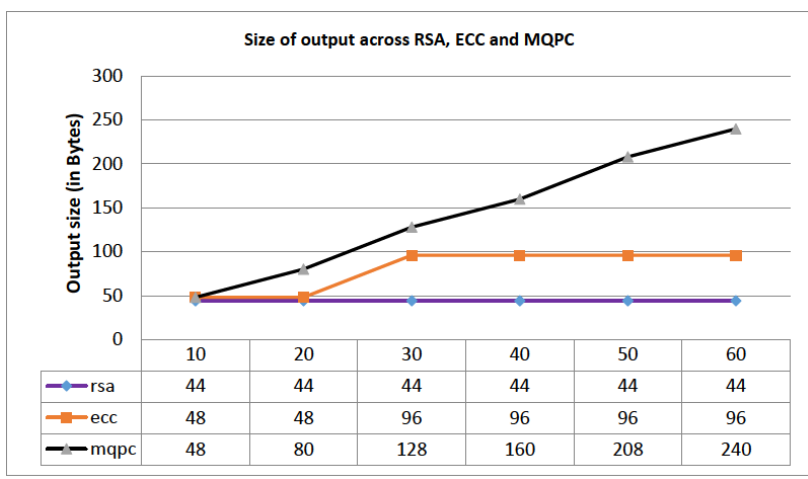

Figure 3: Graph of Size of final outputs of proposed system versus those based on RSA, and ECC.

of output is one of the important properties of serious effect. The larger the size of the output compared with the plaintext, the more work factor required recovering the secret message, and hence, the more secure the information against any Statistical or even Brute-Force attack. In this case, RSA, ECC and our proposed MQPC based secure evoting systems uphold this effect and there are no direct relationships between symbols in the cipher-text to the symbols in the Plaintext (Statistical Analysis).

It can as well be deduced from Figure 3, that our postquantum secure e-voting system is more robust against common attacks like statistical analysis and brute force attacks.

Figure 3 is the graph of Table 2, showing the cumulative size of the eventual output of the proposed system.

\subsection{Throughput}

Throughput here is more like the work done by the system. It is equal to the total plaintext in bytes encrypted and then, embedded in a selected cover-image, divided by the total time taken for that encryption plus embedding procedure.

A system's throughput is directly proportional to its performance. This implies that, the higher the throughput 
Table 3: Throughput of the proposed system across RSA, ECC and MQPC.

\begin{tabular}{cccc}
\hline Size of Input & RSA & ECC & MQPC \\
\hline 10 & 0.0116 & 0.0187 & 0.045 \\
20 & 0.0304 & 0.0702 & 0.0922 \\
30 & 0.0347 & 0.063 & 0.1322 \\
40 & 0.0458 & 0.0926 & 0.1732 \\
50 & 0.0534 & 0.0904 & 0.2146 \\
60 & 0.0614 & 0.1156 & 0.2239 \\
\hline
\end{tabular}

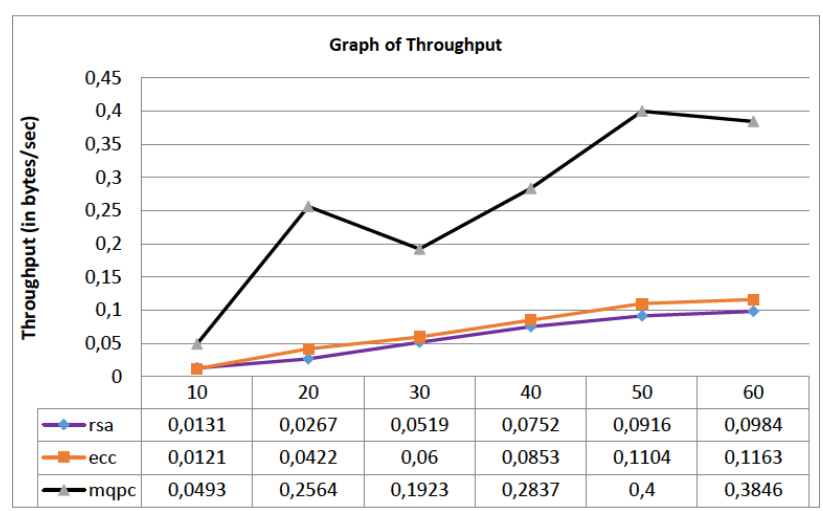

Figure 4: Performance of the proposed system versus those that are based on RSA and ECC, in terms of their throughput.

of a system, the higher the performance of such system. The results obtained on throughput are as shown in Table 3 and plotted in the Figure 4.

\section{Conclusion}

The security of most public-key cryptosystems used in practice depends only on the hardness of solving the factoring and discrete logarithm problems. This fact is enough motivation to study cryptosystems based on other trapdoor one-way functions. After Peter Shor solved in 1994 the two problems mentioned above, the motivation to find an alternative solution that may be used in both classical and quantum computing era even increased. Maybe a large quantum computer will never be constructed and maybe no one will ever solve the factoring and discrete logarithm problem for classical computers. But if this ever occurs, we should be able to have an alternative solution that is secure, efficient and that inspires confidence.

Beyond the aforementioned, there are even more motivations to develop systems that combines the strengths of both cryptography and steganography. The main purpose of this work therefore was to study one (Multivariate
Polynomial based Cryptography) of the four families that are believed to be quantum resistant and based on that, develop a Crystographic electronic voting security framework that integrates Cryptography with Steganography in a 2-step protocol. This chemistry adds multiple layer of security, and satisfy requirements such as capacity, security and robustness for secure data transmission over an open channel, and hopefully motivate further research in these areas.

We have described MQPC based security framework for e-voting systems, also implemented and tested the proposed system. We compared the performance of our electronic voting security system with RSA and ECC based ones, in terms of computation time, size of their outputs and their throughputs. Experimental results show that our proposed secure e-voting system performed better on all grounds. Throughput is the most important parameter that demonstrates the performance of any algorithm. It is observed that the throughput of the proposed MQPC Secure e-voting system is better, compared to the ECC and RSA based ones.

Results have shown that our post-quantum e-voting system has several advantages over ECC and RSA based ones.

Furthermore, we believe that the future of postquantum crypto-systems looks brighter than those of RSA and ECC as post-quantum crypto-systems are believed to be more robust against both classical and quantum attacks.

\section{References}

[1] Hadullo K., Oboko R., Omwenga E., A model for evaluating elearning systems quality in higher education in developing countries, International Journal of Education and Development using Information and Communication Technology (IJEDICT), 2017, 13(2), 185-204

[2] Traxler J., Distance Learning-Predictions and Possibilities Journal of Education Science, 2018, 8, 35; DOI: https://doi:10.3390/ educsci8010035, www.mdpi.com/journal/education

[3] Getachew H. M., Monica J. G., A contextualized IT adoption and use model for telemedicine in Ethiopia, Journal of Information Technology for Development, 2019, 25(2), 184-203, DOI: https: //doi.org/10.1080/02681102.2018.1461057

[4] Al-rawahna A. S. M., Chen S., Hung C., The Barriers Of EGovernment Success: An Empirical Study From Jordan, International Journal of Managing Public Sector Information and Communication Technologies (IJMPICT), 2018, 9(2), DOI: https: //doi//10.5121/ijmpict.2018.9201

[5] Chipeta J., A Review of E-government Development in Africa A case of Zambia, Journal of e-Government Studies and Best Practices, Vol. 2018 (2018), Article ID 973845, 
DOI:10.5171/2018.973845

[6] Li X., He X., Zhang Y., The impact of social media on the business performance of small firms in China, Journal of Information Technology for Development, 2019, 1-23, DOI: https://doi.org/ 10.1080/02681102.2019.1594661

[7] Kuboye B. M., Gabriel A. J., Thompson A. F., Joseph V. O., Analysis of algorithms in long term evolution (LTE) network, Journal of Computer Science and Its Application, 2018, 25(2), 59-71

[8] Pham Q. C., Tran X. P., Misra S., Maskeliunas R., Damaševicius R., Relationship between Convenience, Perceived Value, and Repurchase Intention in Online Shopping in Vietnam, Journal of Sustainability 2018, 10, 156; doi:10.3390/su10010156, www. $\mathrm{mdpi.com/journal/sustainability}$

[9] Gabriel A. J., Egwuche S. O., Modelling the Employees' Activities of Public Service Sector using Production Rules. Anale. Seria Informatică. Vol. XIII fasc. 2 - 2015 Annals. Computer Science Series, University of Timisoara, Romania, 2015, 13(2), 65-68

[10] Iwasokun G. B. Egwuche O. S., Gabriel A. J., Neural NetworkBased Health Personnel Monitoring System, African Journal of Computing \& ICT; IEEE, 2015, 8(1), 79-87

[11] Agboola A. H., Gabriel A. J., Aliyu E. O., Alese B. K., Development of a Fuzzy Logic Based Rainfall Prediction Model, International Journal of Engineering and Technology, 2013, 3(4), 427-435, ISSN: 2049-3444.

[12] Abo-Rizka M., Ghounam H. R., A Novel E-voting in Egypt, International Journal of Computer Science and Network Security, 2007, 7(11), 226-234

[13] Alok K., Atul K., A Novel Approach for Secure Mobile-Voting using Biometrics in Conjunction with Elliptic Curve Crypto-Stegano Scheme, International Journal of Technology and Engineering System (IJTES), 2011, 2(1), 8-11

[14] Cetinkaya O. and Koc M. L, Practical Aspects of DynaVote; Evoting Protocol. Electronic Journal of E-Government, 2009, 7(4), 327-338

[15] Ibrahim S., Kamat M., Salleh M., Abdul-Aziz S., 2003, Secure voting using blind signature available at URL http://eprints.utm. my/3262/1/IEEE02_EVS_full_paper_ver14Nov.pdf, Retrieved on November 17th 2017

[16] Gabriel A. J., Alese B. K., Adetunmbi A. O., Adewale O. S., PostQuantum Crystography based Security Framework for Cloud Computing, Journal of Internet Technology and Secured Transactions (JITST), 2015, 4(1), 351-357
[17] Subashini S. and Kavitha, V., A Survey on Security Minimal issues in service delivery models of cloud computing, Journal of Network and Computer Applications, 2011, 34(1), 1-11

[18] Rura L., Isaac B., Haldar M. K., Secure Electronic Voting System Based on Image Steganography. Proceedings of IEEE Conference on Open Systems (ICOS2011), IEEE, September 25-28, Langwi, Malaysia. 2011

[19] Okediran O. O., Omidiora E. O., Olabiyisi S.O, Ganiyu R. A, Alo 0.O., A framework for a Multifaceted Electronic Voting System, International Journal of Applied Sciences, 2011a, 1(4), 135-142

[20] Alese B. K., Philemon E. D., Falaki S. O. Comparative Analysis of Public Key Encryption Schemes. International Journal of Engineering and Technology, 2012, 2(9)

[21] Gabriel A. J., Alese B. K., Adetunmbi A. O., Adewale O. S., PostQuantum Crystography: A Combination of Post-Quantum Cryptography and Steganography, The 8th International Conference for Internet Technology and Secured Transactions (ICITST-2013), Technically Co-sponsored by IEEE UK/RI Computer Chapter, $9^{\text {th }}$. $12^{\text {th }}$ December 2013, London, UK, 454-457

[22] Olaniyi, O. M., Arulogun O. T., Omidiora E. O., Okediran O. O., A Survey of Cryptographic and Stegano-Cryptographic Models for Secure Electronic Voting System, Covenant Journal of Informatics and Communication Technology (CJICT), 2013, 1(2), 54-73

[23] Rajyaguru M. H., CRYSTOGRAPHY - Combination of Cryptography and Steganography With Rapidly Changing Keys. International Journal Technology and Advanced Engineering Website: www.ijetae.com ISSN 2250-2459, 2012, 2(10)

[24] Daodu M. N., Gabriel A. J. Alese B. K., Adetunmbi A. O., A Data Encryption Standard (DES) based Web Services Security Architecture, Annals of Computer Sscience Series, Tibiscus University, 2016, 14(2), 53-58

[25] Alese B. K., Gabriel A. J., Olukayode 0., Daramola O. A., Modelling of Risk Management Procedures for Cybercrime Control Systems, Proceedings of the World Congress on Engineering (WCE 2014) London, United Kingdom, ISBN: 978-988-19252-7-5

[26] Petzoldt A. (2013). Selecting and Reducing Key sizes for Multivariate Cryptography. PhD. Thesis., Technical University Darmstadt, Germany, 2013

[27] Shor P., Polynomial-time algorithms for prime factorization and discrete logarithms on a quantum computer, SIAM Journal on Computing. 1997, 26(5), 1484-1509 